\title{
Estimativa da entropia em Amparo de São Francisco, Sergipe - Brasil
}

\author{
Estimation of entropy in Amparo de São Francisco, Sergipe - Brazil \\ Estimación de entropía en Amparo de São Francisco, Sergipe - Brasil
}

\section{Resumo}

A proposição da entropia foi empregada para avaliar as oscilações pluviais tentando compreender ou acrescentar mais informações pluviais da região. Avaliar as oscilações dos dados de chuvas diárias, mensais e anuais para Amparo de São Francisco e debater suas disponibilidades hídricas, observando a metodologia da entropia para as quadras secas e chuvosas e seus desvios padrão, entre 1964-2020. As séries pluviais diárias anuais foram proporcionada através da função de distribuição de probabilidade de chuva e a média anual da entropia, sendo calculada para cada ano através das informações dessa média. Utilizou-se a unidade "bit" para o cálculo do elemento em estudo, tendo como significado dígito binário e a menor unidade numérica admitida foram os valores de $0 \mathrm{ou} 1$. O rendimento da informação resulta redução da entropia, e vice-versa. A entropia torna-se zero quando existe certeza absoluta da ocorrência de um certo evento, ou estatisticamente, quando todas as probabilidades do conjunto, exceto uma, é zero.

Palavras-chave: Desordem energética; Flutuações diárias pluviais; Distribuição pluvial.

\begin{abstract}
The entropy proposition was used to assess the fluctuations in rainfall, trying to understand or add more rainfall information about the region. Evaluate the fluctuations of daily, monthly and annual rainfall data for Amparo de São Francisco and discuss their water availability, observing the entropy methodology for dry and rainy blocks and their standard deviations, between 1964-2020. The annual daily rainfall series were provided through the rainfall probability distribution function and the annual average of entropy, being calculated for each year through the information of this average. The "bit" unit was used to calculate the element under study, meaning binary digit and the smallest numerical unit accepted were the values of 0 or 1 . The yield of information results in a reduction in entropy, and vice-versa. Entropy becomes zero when there is absolute certainty that a certain event will occur, or statistically, when all but one probability in the set is zero.
\end{abstract}

Keywords: Energy disorder; Rainfall daily fluctuations; Rain distribution.

\section{Resumen}

La proposición de la entropía se utilizó para evaluar las fluctuaciones en las precipitaciones, tratando de comprender o agregar más información sobre las precipitaciones sobre la región. Evaluar las fluctuaciones de los datos de precipitación diaria, mensual y anual para Amparo de São Francisco y discutir su disponibilidad de agua, observando la metodología de entropía para bloques secos y lluviosos y sus desviaciones estándar, entre 1964-2020. Las series de precipitación diaria anual se proporcionaron a través de la función de distribución de probabilidad de lluvia y el promedio anual de entropía, siendo calculado para cada año a través de la información de este promedio. La unidad de "bit" se utilizó para calcular el elemento en estudio, es decir, dígito binario y la unidad numéricas más pequeña aceptada fueron los valores de 0 o 1 . El rendimiento de información da como resultado una reducción de la entropía y viceversa. La entropía se 
vuelve cero cuando existe la certeza absoluta de que se producirá un determinado evento, o estadísticamente, cuando todas las probabilidades menos una del conjunto son cero.

Palabras clave: Trastorno energético; Fluctuaciones diarias de las precipitaciones; Distribución de lluvia.

\section{Introdução}

A entropia relativa e a variabilidade sazonal podem simular a eficácia do desenvolvimento vegetativo. Segundo Souza, Feng, Antônio, Montenegro e Porporato (2016), o desenvolvimento da caatinga replica o total pluvial, assim como a distribuição da chuva ao longo da sazonalidade.

O estudo da desordem e o desafio da análise não seguindo uma sequência linear, ou ao menos previsível como os fenômenos atmosféricos extremos, nos leva a compreender as variabilidades climáticas instigantes.

Nem mesmo a ciência é livre de contestações e subversões, é só olharmos para o desaparecimento do determinismo científico para o surgimento de uma época de maior iluminismo científico. Pontes (2016) fez uma extensa referência em seu texto às observações de Blumenfeld (1981), sobre abaixamento da entropia geral nas organizações celulares para formação de moléculas complexas que dão origem a vida como a conhecemos, chegando a afirmar que a formação de estruturas biológicas é feita sobre tudo, livre de um custo termodinâmico considerável.

Araújo (2017) no seu estudo sobre a entropia na construção de softwares na inteligência artificial e na árvore de decisão, levando em consideração os parâmetros do ambiente: aspecto do céu; vento; temperatura e umidade e demonstrou a significância da entropia no referido estudo devido a sua variabilidade atmosférica.

Ferreira, Silva, Stogic, Meneses, Irmão e Souza (2018) analisaram a oscilação espacial da dinâmica pluvial no estado de Pernambuco aplicando a metodologia da entropia. Aplicou-se o método nas séries temporais, considerando-se as correlações temporais entre os dados da série por ter representação simbólica baseada nas comparações dos seus valores. Os resultados mostraram valores da entropia reduzidos no setor do litoral, indicando maior variabilidade e menor previsibilidade das chuvas mensais para as regiões do litoral, zona da Mata e Agreste. Nas regiões do sertão, registrou-se menor variabilidade e maior previsibilidade das chuvas.

Medeiros (2019) aferiu a avaliação da entropia para as cidades São Bento do Una, Serra Talhada e Caruaru (PE), referentes aos anos 2012, 2016, 2008 e 2011, para as fases dos fenômenos El Niño e La Niña levando em consideração as quadras secas e chuvosas. Os resultados revelaram que as chuvas estão conectadas as irregularidades e as oscilações meteorológicas de microescala local nos quais ocasionaram chuvas inferiores à média climatológica na área estudada. Em São Bento do Una e em Serra Talhada, o fenômeno El Niño e La Niña, não registaram aumentos ou redução das chuvas. Os cenários pluviométricos incorporados às oscilações espaciais e temporais mostraram que a classificação e a regionalização climática formam acertos aos modelos de previsão em uso no Brasil.

Medeiros (2019) avaliou as oscilações pluviais e discutiu a disponibilidade hídrica, através do procedimento da entropia para as quadras secas e chuvosas levando em consideração as flutuações dos desvios padrão entre 1962-2015 para Recife - PE, tendo em vista compreender as particularidades das dinâmicas pluviais em estudo. O autor mostrou que a cidade de Recife - PE nas fases da La Niña não registrou influência nos índices pluviais. O desvio padrão oscilou entre os dados da entropia média.

Gleiser (2016) afirmou que de forma lógica e natural, todos os organismos vivos necessitam de certa forma, capturar certa quantidade de energia externa e transformá-la em "energia interna”, que fomentará a manutenção de seu metabolismo vital, atribuída à figura da alimentação.

Melo e Medeiros (2016) avaliaram a estimativa da entropia, para os padrões espaciais tentando compreender as características pluviais em Cabaceiras - Paraíba. Ressaltaram que o desvio padrão anual flutuou nos contornos da estimativa média da entropia. 
Medeiros, Silva e Gomes Filho (2015) analisaram a pluviometria (1930-2010) para Teresina (PI), levando em conta a estimativa da entropia e dos padrões espaciais que permitem envolver as flutuações pluviais da região. O desvio padrão oscilou uniformemente em relação aos dados da entropia, revelando oscilações nos seus dados em torno da média. A flutuação dos padrões nos índices pluviais foi máxima nas fases de La Niña do que nas faces de EL Niño com índices de entropia anual de 2,68 bits (mínimo) e 6,47 bits (máximo) com valor médio de 5,39 bits.

Para Noronha, Hora e Silva (2016) mostrou que os episódios de secas prolongadas em consequências das mudanças climáticas, tem precisão de um melhor entendimento e de previsões melhoradas para que possam detectar suas ocorrências repentinas.

Holanda, Medeiros e França (2020a) em seus estudos de avaliação às oscilações das entropias das chuvas nos períodos diário, mensal, anual e dos desvios padrão para os períodos secos e chuvosos em Bom Jesus Piauí (1960-2018). Os autores completaram que as altas incidências da entropia foram registradas com chuvas de alta magnitude e valores de entropias baixos em anos de menores índices pluviais.

Objetiva-se o estudo, avaliar as oscilações pluviais diários, mensais e anuais para Amparo de São Francisco (SE) e debater suas disponibilidades hídricas, lembrando a metodologia da entropia pluvial dos períodos secos e chuvosos e dos seus desvios padrão, entre 1964-2020.

\section{Material e Métodos}

Amparo de São Francisco localiza-se na região nordeste de Sergipe em uma região caracterizada por duas estações bem definidas, um período chuvoso oscilando de fevereiro a agosto e um período seco de setembro a janeiro. O clima é do tipo "As" (quente e úmido Tropical chuvoso), segundo a classificação de Köppen (1928); Köppen et al., (1931), esta classificação também foi determinada pelos autores (Medeiros et al, 2019; Alvares et al., 2014).

A Estimativa da entropia da chuva, foi efetivada em planilhas eletrônicas após ajustes de falhas, consistência e homogeneização da série diária pluvial de 1963-2020 aplicando-se as equações

A série dos dados diários pluviais anuais foi delineada pela distribuição de probabilidade pluvial e a média da entropia anual sendo calculada para cada ano através da informação da entropia, obtida pela Equação 1 (Shannon, 1948):

$$
H=-K \sum P_{i} \log P_{i}
$$

Onde:

Pi probabilidade da enésima variável discreta,

$\mathrm{k}$ é constante positiva e depende das unidades trabalhadas, e

H é a entropia da variável aleatória.

Considerando $\mathrm{K}$ constante, como unidade de estimativa, igual a 1 e a base do logaritmo 2, usando-se a equação simplificada:

$H=-\sum_{i=1}^{n} P_{i} \log _{2} P_{i}$

Onde:

H é adquirido em "bit", e n é o número de eventos discreto. 
Utilizaremos a unidade "bit" na estimativa da entropia, tendo como significado dígito binário, constituindo a menor unidade na notação binária assumindo 0 ou 1 como valor.

Se todos os Pi's são iguais, tem-se que, $\mathrm{Pi}=1 / \mathrm{n}$, portanto a entropia é $\mathrm{H}=\log _{2} \mathrm{n}$. Sendo assim, $\mathrm{H}$ é uma função notadamente crescente em n. Para um determinado dado n, H é máximo quando todos os Pi’s são iguais. Ao contrário, H é mínimo e igual a zero quando todos os Pi’s, exceto um, é zero. Resulta que as variáveis aleatórias são sempre as mesmas, portanto, um dos Pi's torna-se unitário. Assim, o valor da entropia, varia dentro do intervalo de zero a $\log 2$ n, de acordo com a forma da distribuição de probabilidade dos Pi’s. O valor da entropia decresce com o aumento do número de contraste e aumenta com o decréscimo desse número. Visto dessa maneira, a entropia pode ser considerada como uma estimativa funcional da incerteza associada à distribuição de probabilidade.

Na série pluvial de um ano, admite-se que $\mathrm{r}_{\mathrm{i}}$ representa a pluviometria diária correspondente ao enésimo dia do ano. Para os índices diários pluviais de 1 de janeiro a 31 de dezembro do mesmo ano serão expressos por $\mathrm{r}_{1}$ e $\mathrm{r}_{365}$. Portanto, a pluviometria total anual não bissexta $\mathrm{R}$ é expressa pelo somatório dos valores diários, variando de $\mathrm{i}=1$ até $\mathrm{i}=365$, aplicando a Equação 3:

$\mathrm{R}=\Sigma \mathrm{r}_{\mathrm{j}}$

Onde:

Os valores de ri podem ser zero para alguns dias e diferentes de zero para outros.

As séries pluviais constituídas por $\mathrm{r}_{1}, \mathrm{r}_{2} \ldots, \mathrm{r}_{\mathrm{n}}$ podem ser fixadas como a frequência de ocorrência acumulada das chuvas para 1,2 , n enésimo dia do ano.

Portanto a frequência relativa da chuva (Pi) consiste em ser obtida dividindo-se ri pelo tamanho total da amostra (R), usando a Equação 4:

$P_{i}=\frac{r_{j}}{R}$

A frequência relativa $(\mathrm{Pi})$ é fixada como função de probabilidade do total pluvial no enésimo dia, e sua distribuição concebe a característica probabilística da partição temporal pluvial ao longo do ano, como sendo a ocorrência da incerteza pluvial, empregando a Equação 5.

$H=-\sum_{i=1}^{n} \frac{r_{i}}{R} \log _{2}\left(\frac{r_{i}}{R}\right)$

$\mathrm{O}$ índice $\mathrm{H}$ é independente da ordem sequencial na série temporal; assume o valor zero quando $\mathrm{R}$ ocorre apenas uma vez no ano e o valor máximo (log2 n) quando R ocorre em todos os dias.

A entropia aproxima-se do seu valor máximo quanto mais uniforme for a sua distribuição, portanto, quando os dados da série exibem pouca variabilidade temporal. Logo, $\mathrm{H}$ pode ser uma estimativa da variabilidade pluviométrica na direção da escala. Quando as séries anuais pluviais para n anos estão disponíveis, em um mesmo posto pluviométrico, a melhor estimativa da entropia deste posto pode ser obtida através da média aritmética e expressa pela Equação 6: 
$\bar{H}=\frac{1}{n} \sum_{i=1}^{n} H$

Onde:

$\mathrm{H}$ entropia média e

n o número de anos para a estimativa de $\mathrm{H}$.

\section{Resultados e Discussões}

Na Figura 1 têm-se as flutuações pluviais anuais e climatológicas dos anos de 1964-2020 para o município de Amparo de São Francisco - Sergipe. Com média histórica de 1130,4 mm. Os altos índices pluviais registraram-se nos anos de 1964 a 1967, 1973. As menores pluviometrias ocorreram em 1970, 1980, 1993, 2012, 2016 e 2018. As contribuições locais e regionais; formações de linhas de instabilidades; atividades da Zona de Convergência do Atlântico Sul contribuíram para as variabilidades pluviais discutidos na Figura 1. Estas variabilidades corroboram com os estudos de (Alveres, Stape, Sentelha, Gonçalves \& Sparovec 2015; IPCC 2014; Holanda \& Medeiros 2020b; Maraengo, Alves, Beserra \& Lacerda 2015).

Figura 1. Precipitação anual e climatológica em Amparo de São Francisco - Sergipe.

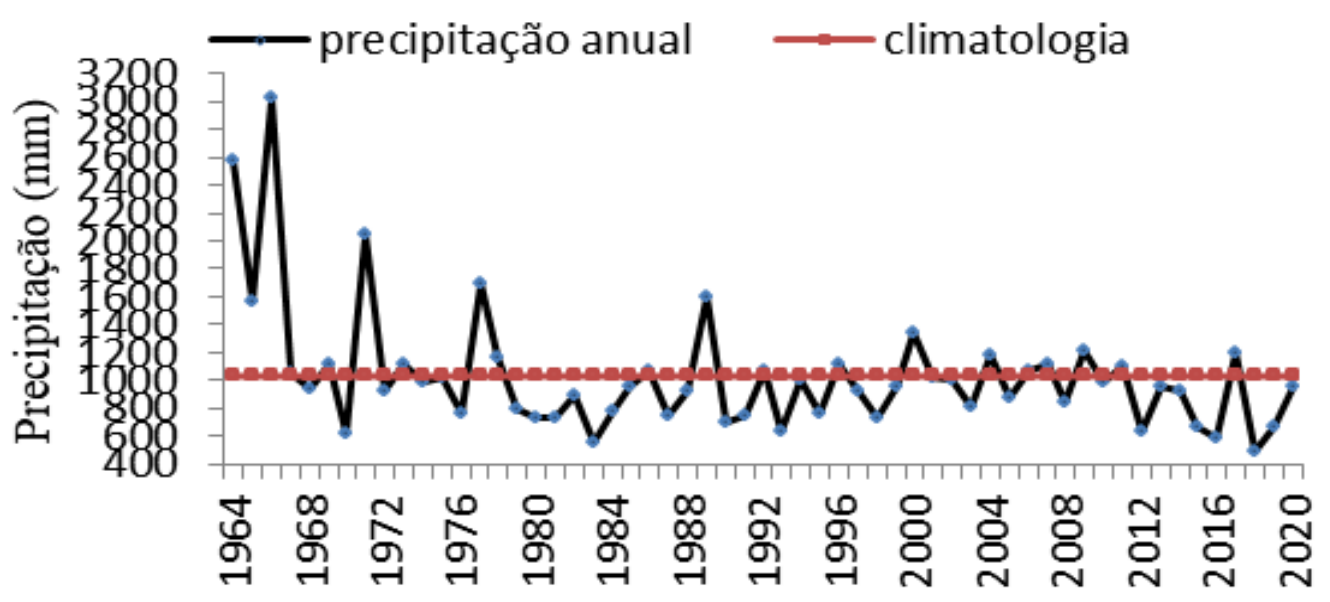

Fonte: França (2021).

Estudos apontam que a variabilidade temporal das chuvas é de suma importância para quantificar as consequências ocasionadas na disponibilidade d'água no solo, escoamento superficial e erosão, desordem pluvial como enchentes e alagamentos em áreas isoladas. (Nadarajah \& Cheid 2007; Westra, Alexandre \& Zwiers 2013).

As flutuações pluviais dos períodos chuvosos e secos para Amparo de São Francisco estão demonstradas na Figura 2. As magnitudes pluviais registradas no período chuvoso foram auxiliadas pelas atividades da Zona de Convergência do Atlântico Sul, acoplamentos de entradas de frentes frias, contribuição das formações de linhas de instabilidade, vórtices ciclones de altos níveis e orografia (Medeiros, 2020).

Os anos de 1964, 1966, 1971; 1978; 1998 registraram os maiores anos com cotas pluviais elevadas, os anos de 1970; 1980; 1993; 2016 e 2018 as menores pluviometrias da quadra chuvosa. Observam-se ainda as irregularidades registradas para os demais anos em estudos. No período seco registraram-se eventos extremos pluviais nos anos de 1966; 1971 e 2004 ocasionados pela troca de calor e a alta umidade relativa. Nos demais anos as chuvas ocorridas não foram suficientes para repor a capacidade de campo do período seco. Estudos com similaridades de índices pluviais irregulares para o período seco foram detectados pelos autores (Menezes, Medeiros, Neto \& Menezes 2015). 
Figura 2. Distribuição pluvial do período chuvoso e seco em Amparo de São Francisco - Sergipe.

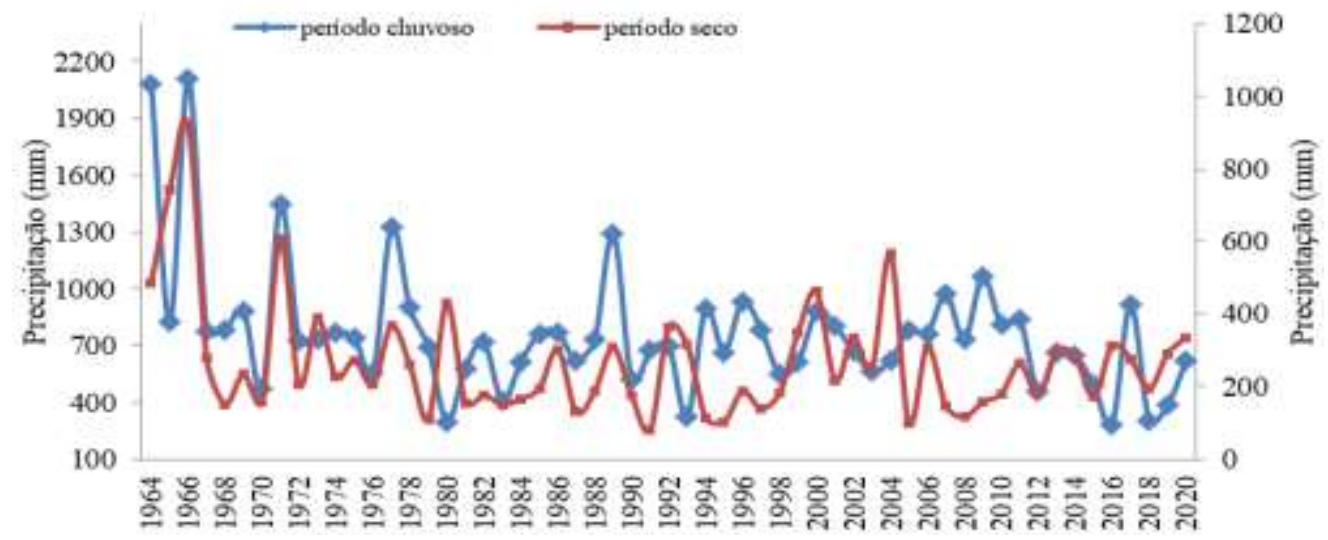

Fonte: França (2021)

As oscilações anuais dos desvios padrão normalizadas dos índices pluviais em Amparo de São Francisco - Sergipe no período de 1964 - 2020 podem ser destacadas na Figura 3. As flutuações dos desvios negativos ocorreram fluíram entre -1,5 mm (1975; 2003 e 2010), nos anos de 1986 a 1998 registrou-se as maiores oscilações pluviais negativas. Os anos de 164; $1966 ; 1971$ e 1977 ocorreram os maiores desvios normalizados positivos, nos demais anos de desvios positivos as flutuações oscilaram entre 0,5 mm a 32,5 mm. O estudo de Medeiros et al, (2020) corroboram com os resultados em discursões.

Figura 3. Distribuição do desvio normalizado da precipitação anual em Amparo de São Francisco - Sergipe.

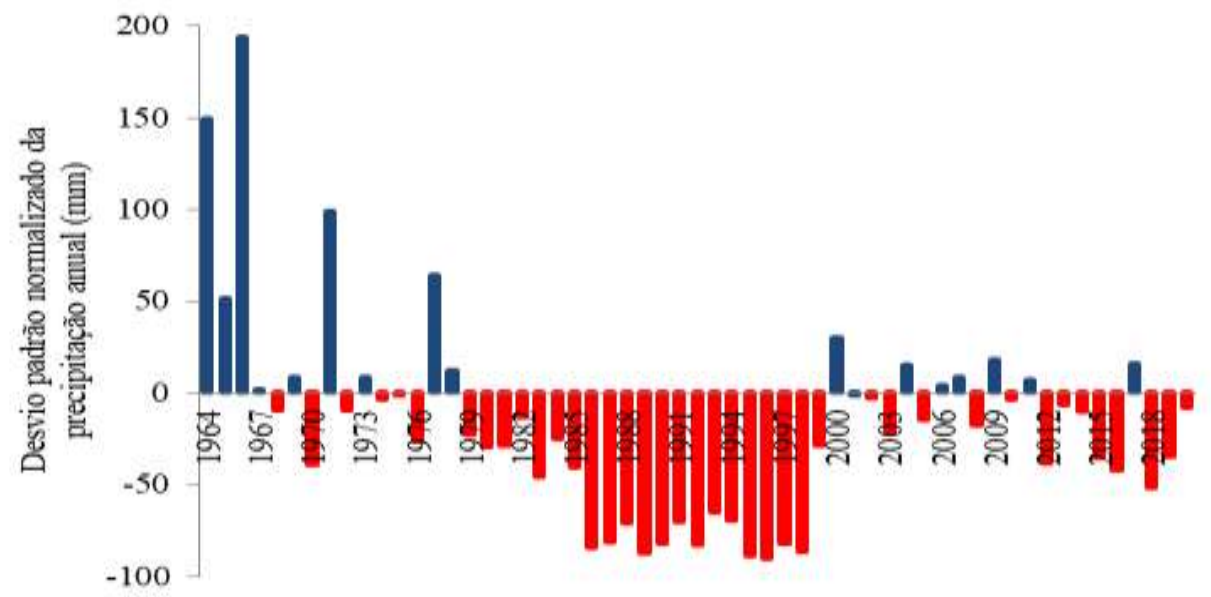

Fonte: França (2021).

A incerteza pode ser quantificada pela entropia, levando-se em conta todos os tipos de informações disponíveis e representada pela distribuição de probabilidade da variável controlada. Interpretando um conjunto composto por n elementos e se considerando pi como a probabilidade de encontrar o sistema no enésimo microestado, a entropia de Shannon é igual a entropia da mecânica estatística, (Silva 2004).

Na Figura 4 tem-se o percurso anual da entropia pluvial do período de 1964 a 2020. Destaca-se comportamento irregular na flutuação da entropia anual no decorrer do período estudado, os anos de 1964, 1965, 1967, 1970, 1981, 1987, 1993, 1999 onde se registrou as baixas incidências da entropia. Os anos 1971, 1972, 1975, 1976 como os de maiores flutuações da entropia. Salienta-se que nos demais anos de estudo, os valores da entropia oscilaram entre 6,2 bits a 5,2 bits. Ressalta-se que a entropia tolera redução de seus valores quando acontecem menores registros pluviais, admitindo deste modo as variabilidades de ocorrências das chuvas na área estudada. O ganho de uma informação resulta no decréscimo e/ou acréscimo do índice da entropia. 
A entropia torna-se zero quando existe certeza absoluta da ocorrência de certo evento, ou estatisticamente, quando todas as probabilidades de um conjunto, exceto uma, é zero. Estudos como os dos autores, (Melo \& Medeiros 2016) corroboraram com os resultados discutidos.

Figura 4. Entropia anual e histórica em Amparo de São Francisco do período de 1964-2020.

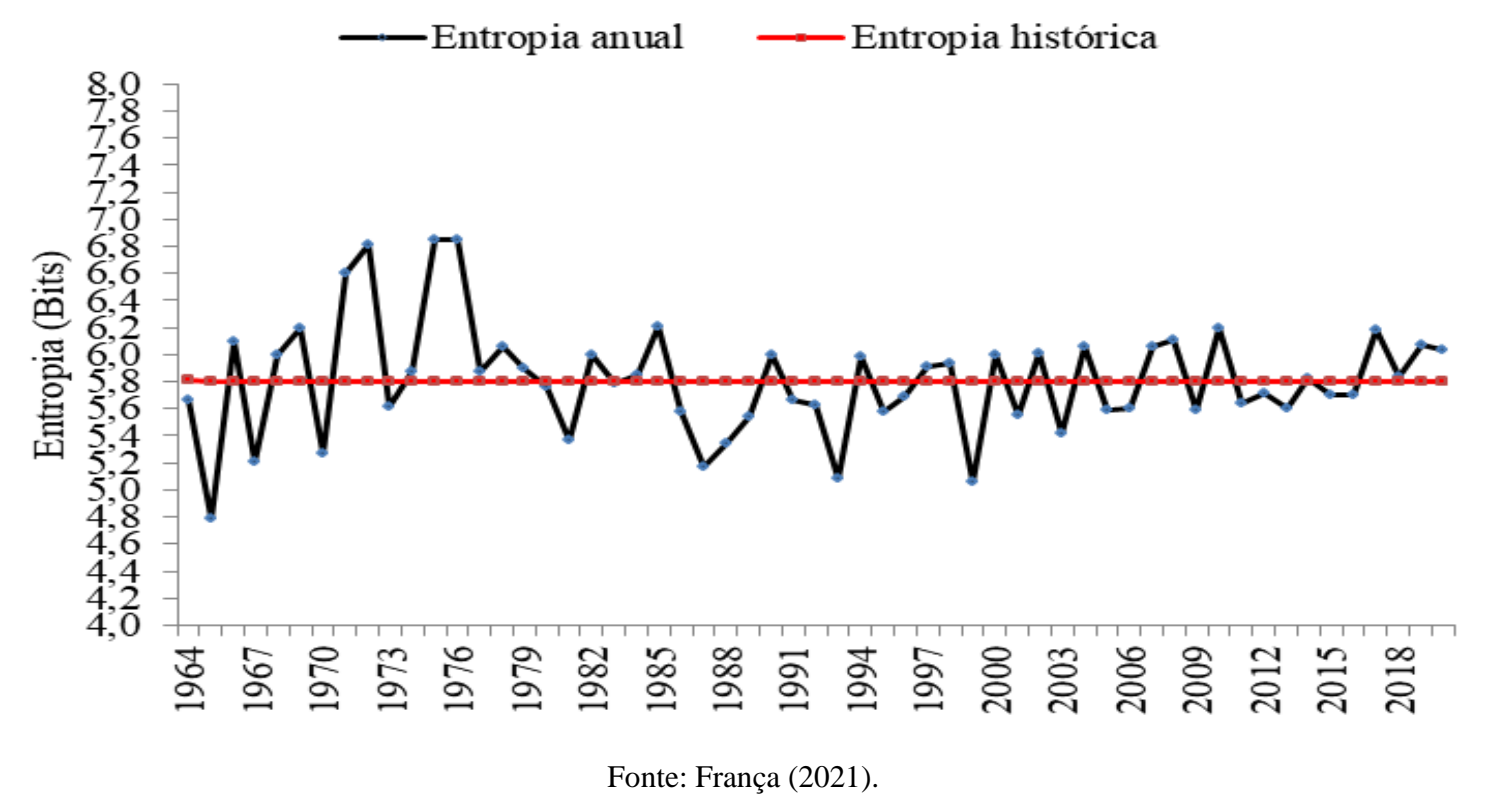

Na Figura 5 tem-se a variabilidade da entropia do período chuvoso e seco em Amparo de São Francisco do período de 1964-2020. No período chuvoso a entropia oscilou entre 2,8 bits a 5,8 bits. Destaca-se os anos de alta entropia dentro o período chuvoso 1972 (5,8 bits); 1976 (5,3 bits); 1995 (5,4 bits) e 2007 (5,6 bits). Os anos com baixos valores de entropia registrados entre os períodos chuvosos foram: 1965 (3,3 bits); 1980 (2,8 bits); 1993 e 2004 (3,3 bits); 2015 (3,0 bits). 
Figura 5. Entropia do período chuvoso e seco em Amparo de São Francisco do período de 1964-2020.

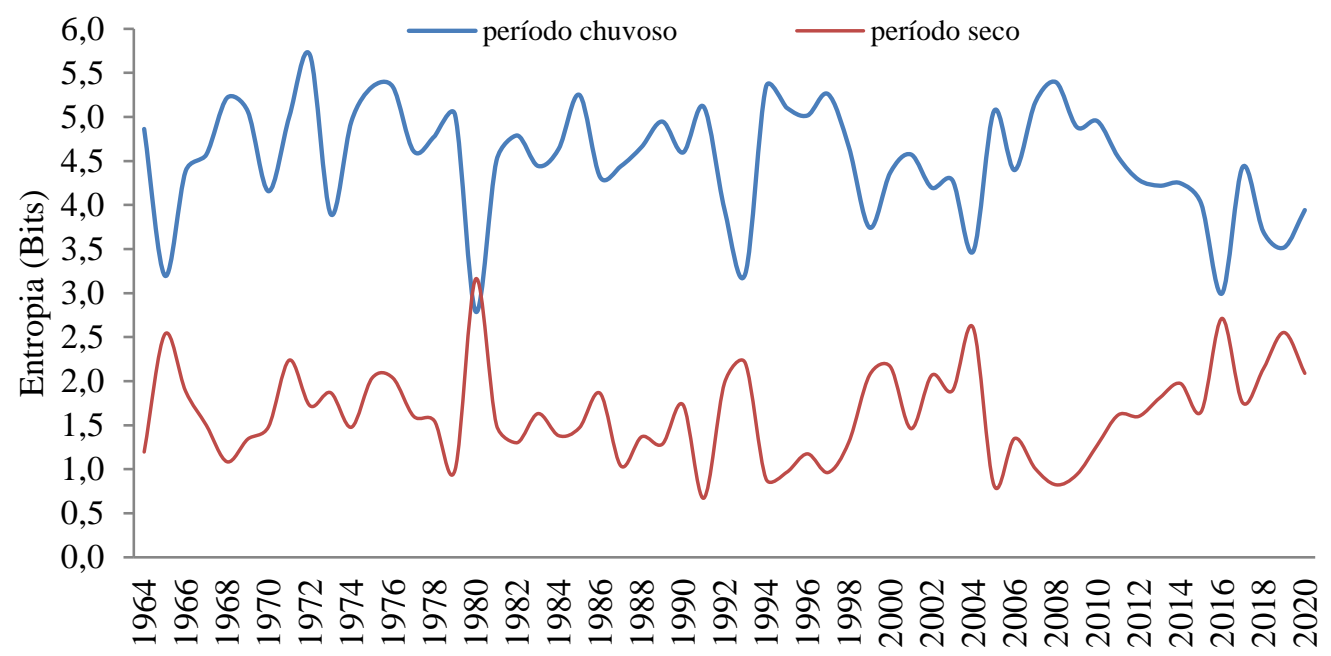

Fonte: França (2021).

O período seco da entropia (Figura 5) nos mostram as suas variabilidades fluindo entre 3,3 bits (1981 a 0,7 bits (1991). Observando o gráfico do período seco destaca-se os maiores valores da entropia para os anos 1965 (2,5 bits); 1981 (3,3 bits); 1993 (2,7 bits); 2003 (2,6 bits) e os anos de 2015 e 2019 com 2,7 e 25 bits respectivamente. Os menores valores da entropia para o período seco registraram-se nos anos de 1991 (0,8 bits); 1994 (0,9 bits); 2005 (1,0 bits) e 2007 (0,8 bits). Estudos que corroboram com os resultados destes estudos podem ser visto em (Medeiros 2019; Melo \& Medeiros 2019).

Na Figura 6 tem-se a distribuição do desvio normalizado da entropia anual em Amparo de São Francisco - Sergipe. O desvio padrão normalizados da entropia anual negativa oscilou entre -17 bits a -1 bits. Os anos de maiores desvios negativos foram 1980; 2012; 2015 e 2016. Os menores desvios neg5ivos ocorreu nos anos de 1965; 1993 e 1999.

Os maiores desvios positivos registraram-se nos anos: 1972; 1975; 1976 e os menores desvios positivos ocorreram nos anos de 1984; 2014 e 2018. Os estudos de (Melo \& Medeiros 2019; Medeiros 2019) corroboram com as discussões apresentadas.

Figura 6. Distribuição do desvio normalizado da entropia anual em Amparo de São Francisco - Sergipe.

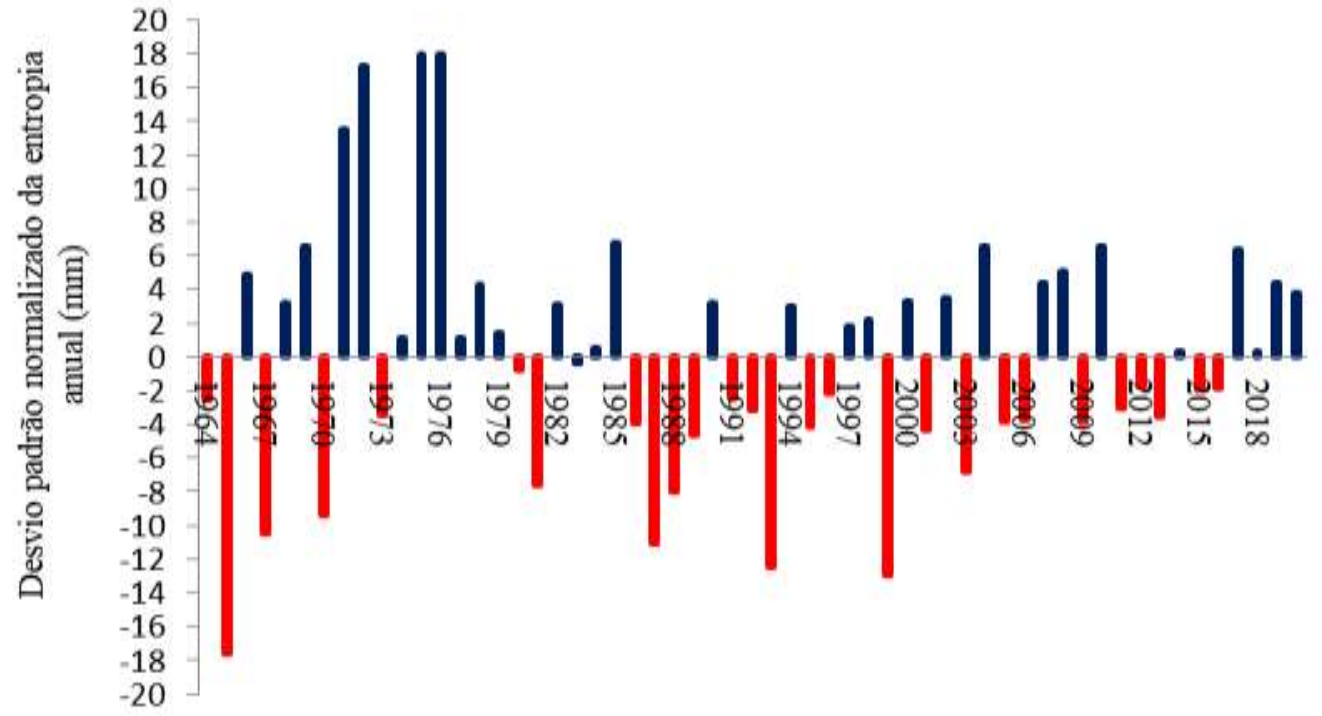

Fonte: França (2021). 
Research, Society and Development, v. 10, n. 15, e389101522800, 2021

(CC BY 4.0) | ISSN 2525-3409 | DOI: http://dx.doi.org/10.33448/rsd-v10i15.22800

\section{Conclusões}

A técnica da entropia se constitui em ferramenta apropriada para expressar a variabilidade de dados em torno da média, em comparação com a técnica convencional do desvio padrão.

O desvio padrão da entropia anual varia com os valores de entropia, demostrando oscilação de seus dados em torno dos valores médios.

O estudo fornece informações de aplicações da entropia em diversos setores e os autores indicam estes estudos para serem aplicados em estudos de erosão fluvial.

\section{Referências}

Alvares, C. A., Stape, J. L., Sentelhas, P. C., Gonçalves, J. L. M. \& Sparovek, G. (2014). Climate classification map for Brazil. Meteorologische Zeitschrift. 22, $711-728$

Araújo, J. M. F. R. (2017). Inteligência artificial e árvore de decisão utilizando-se da entropia. Universidade Federal de Campina Grande. Departamento de Sistema e Informativa 18p.

Ferreira, D. V. S., Silva, A. S. A., Stosic, T., Menezes, R. S. C., Irmão, R. A., \& Souza, W. S. (2018). Análise da variabilidade espaço temporal da chuva mensal no Estado de Pernambuco utilizando o método entropia de permutação. Revista Brasileira de Biom. 36(2), $276-289$.

Gleiser, M. (2016). Universo eterno, vida eterna? Para se adaptar ao aumento de entropia, a vida terá que mudar. Brasil Escola. http://Brasilescola.uol.com.br/fisica/a-lei-hubbleexpansão- universo.htm.

Holanda, R. M., Medeiros, R. M., \& França, M. V. (2020). Estimativa da entropia pluvial em Bom Jesus Piauí, Brasil. Research, Society and Development, 9(8), e794986010, 2020. http://dx.doi.org/10.33448/rsd-v9i8.6010

Holanda, R. M \& Medeiros, R. M. (2020). Comportamento térmico e a contribuição pluvial em Lagoa Seca, Brasil entre 1981-2019. Research, Society and Development. 9, 695974815 - 30,

IPCC. (2014). Impacts, Adaptation, and Vulnerability. Part B: Regional Aspects. Working Group II Contribution to the Fifth Assessment Report of the Intergovernmental Panel on Climate Change. Intergovernmental Panel on Climate Change (IPCC).

Marengo, J., Alves, L. M., Beserra, E. A., \& Lacerda, F. F. (2015). Variabilidade e mudanças climáticas no semiárido brasileiro. Recursos hídricos em regiões áridas e semiáridas.

Medeiros R. M. (2020). Estudo Agrometeorológicos para o Estado de Sergipe. Distribuição avulsa. P.132.

Medeiros, R. M., França, M. V., Saboya, L. M. F., Holanda, R. M., Rolim Neto, F. C., \& Araújo, W. P. (2020). Análise estatística das precipitações de Serra Talhada e São Bento do Una - Pernambuco, Brasil. Research, Society and Development. 9, e3909119954.

Medeiros, R. M., Silva, V. P. R., \& Gomes Filho, M. F. (2015). Aplicação da teoria da entropia no estudo da precipitação em Teresina-PI. Revista de Geografia, 32(2), 206-218.

Medeiros, R. M. (2019). Variabilidade da entropia pluvial entre os municípios São Bento do Una, Serra Talhada e Caruaru (Pernambuco - Brasil) em período de el niño (a). Revista Equador, 8(1), 116-132.

Medeiros, R. M. (2019). Entropia pluviométrica na grande metrópole Recife-PE, Brasil. Journal of Environmental Analysis and Progress. 04(01), 031-047.

Melo, V. S., \& Medeiros, R. M. (2016). Entropia da precipitação pluvial no município de Cabaceiras - PB, Brasil. Revista Brasileira de Agricultura Irrigada, $10(5), 952-964$

Nadarajah, S., \& Choi, D. (2007). Maximum daily rainfall in South Korea. Journal of Earth System Science, 116, 311-320.

Noronha, G. C. D., Hora, M. D. A. G. M., \& Silva, L. P. D. (2016). Rain Anomaly Index Analysis for the Santa Maria/Cambiocó Catchment, Rio de Janeiro State, Brazil. Revista Brasileira de Meteorologia,31(1), 74-81.

Pontes, J. (2015). Determinism, chaos, selforganization and entropy. Anais da Acad. Bra. de Ciências, 185.

Silva, V. P. R. (2004). Entropy on climate variability in Northeast of Brazil. Journal of Arid Environments, 58(4), 575-596.

Souza, R., Feng, X., Antonino, A., Montenegro, S., Souza, E., \& Porporato, A. (2016). Vegetation response to rainfall seasonality and interannual variability in tropical dry forests. Hydrological Processes, 30(20), 3583-3595.

Westra, S., Alexander, L. V., \& Zwiers, F. W. (2013). Global in creas ingtrends in annual maximum daily precipitation. Journal of Climate, 26(11), 3903-3918. 